\title{
LOW-COST, INTELLIGENT DATA ACQUISITION SYSTEM FOR QCM AND OTHER RESONATOR-BASED BIO- AND CHEMICAL SENSORS
}

\author{
Sergey Y. Yurish \\ Universitat Politècnica de Catalunya (UPC-Barcelona), \\ Centre de Disseny d'Equips Industrials (CDEI-UPC) \\ C/Llorens Artigas, 4-6, planta 0, Edifici U, Campus Sud, 08028 Barcelona, Spain, \\ e-mail: syurish@sensorsportal.com \\ Web address: www.sensorsportal.com
}

\begin{abstract}
A low-cost, intelligent data acquisition system for quartz crystal microbalance (QCM) and other (superficial SAW and bulk acoustic wave) resonator-based chemical and biosensors described in this article. The system is based on the novel modified method of the dependent count and allows obtaining high metrological performances: programmable and constant in all frequency range relative error of measurement up to 0,0005 \% (5 ppm); nonredundant, reduced conversion time of measurement and wide frequency range from $0.05 \mathrm{~Hz}$ to $9 \mathrm{MHz}(144 \mathrm{MHz}$ with prescaling). The developed system allows measuring variations of the resonance frequency shift for QCM sensors working in liquids and gases. The described low-cost data acquisition system is based on novel integrated circuit of universal frequency-to-digital converter. This design approach has eliminated much of the hardware: there is no need for a microcontroller and complex sensor interfacing electronics, as it is directly compatible with PC computers.
\end{abstract}

Keywords: data acquisition system, sensors, method of the dependent count, frequency-to-digital converter, UFDC-2.

\section{INTRODUCTION}

Many modern "electronic noses", "tongues" and various precision applications for analytical chemistry are based on different chemical, mass variation sensors: quartz crystal microbalance (QCM), superficial SAW and bulk (BAW) acoustic wave sensors [1-4]. All of these are built-up on a quartz piezoelectric substrate. Most gravimetric biosensors and immunobiosensors also use piezoelectric quartz crystals either as resonating crystals, or as bulk/surface acoustic wave devices [58].

For quartz piezoelectric crystal (AT-cut) the crystal resonance frequency changes by $\Delta f$ when a mass change $\Delta m$ occurs on the crystal according to the Sauerbrey equation [6]:

$$
\Delta f=-2 \cdot f_{0}^{2} \frac{\Delta m}{A \cdot\left(\mu_{q} \cdot \rho_{q}\right)^{1 / 2}}=-k \cdot \Delta m
$$

where $f_{0}$ is the fundamental frequency of the quartz crystal, $\Delta m$ is the mass change, $A$ is the piezoelectrically active area, $\rho_{q}$ is the density of quartz $\left(2.648 \mathrm{~g} \cdot \mathrm{cm}^{-3}\right), \mu_{q}$ is the shear modulus $\left(2.947 \times 1011 \mathrm{dyn} \cdot \mathrm{cm}^{-2}\right.$ for AT-cut quartz $)$ and $k$ is the constant, which is determined by the quartz crystal type. The change in resonant frequency $(\Delta f)$ in $\mathrm{Hz}$ is directly proportional to the mass adsorbed on the crystal surface. As usually, a crystal has a characteristic resonance frequency $\left(f_{0}\right)$ in the $\mathrm{MHz}$ range, whereas a deposition causes a shift $(\Delta f)$ of the resonance frequency up to a few hundred of ppm [1].

There are several methods and systems for the sensor resonance frequency shift measurement, reported in literature. E.g. the methods of impedance analyze, mentioned in [1] based on calculation of the response frequency variation on the basis of the impedance variation by mean of a vectorial impedance meter; the method of impedance analyze at which the amplitude-frequency characteristic is determined that is shifted when the resonance frequency is shifted [9]. An advance of such methods is a possibility to measure also a quartz crystal quality factor together with the resonance frequency. The main demerit is complex interface electronics. There is also a method of a frequency variation measurement via analog phase measurements [1] with the same demerit. Some other methods are based on digital signal processing (DSP) [9]. For example: 1) Methods based on spectral function analysis with the help of fast 
Fourier transform (FFT). The advantage of this method is simple realization based on DSP microprocessor, a disadvantage - low accuracy. 2) Most likelihood methods based on a signal approximation by the sine curve set or by the use of rejection filters. These methods have a high accuracy of frequency estimation but they need complex calculations. 3) Methods based on analyze of autocorrelation matrix of signal. Such method has a high accuracy, but there are strong requirements to the signal duration. 4) Interpolated FFT based methods, which also have a demerit - low conversion speed due to the necessity of some hundred periods processing.

Most suitable methods for the sensor resonance frequency shift measurement are so-called active (or self-excited oscillator) methods. A QCM sensor is used in a high frequency generator circuit as a stabilization element. The frequency of generator is changing according to the sensor resonance frequency [9]. Main difficulties of data acquisition systems design for such QCM sensors are high frequency signal digitizing, which require expensive ADCs. It is necessary, at first, to shift a high frequency signal to low (some $\mathrm{kHz}$ ) frequency range. An example of such system is described in [9]. This circuit solution is relatively complex and requires an ADC, DSP microprocessor, for example, ADS21XX from Analog Devices, and specialized custom made software for digital filtering, interpolation FFT, etc. [9].

In addition, there are some limitations for the input signal and its frequency range. These mean that the frequency range is more or less determined, and measurand frequency is inside this frequency range. In other words, the frequency range, which contains a precise frequency value, should be known before [9]. As it was reported in [9] the measuring system based on the described method and circuit solution lets receive the absolute error $\pm 0.1 \mathrm{~Hz}$ at measurement of $1754 \mathrm{~Hz}$. This corresponds to $0.0057 \%$ relative error. The time of measurement is 0.1 s. However, as it was shown in [1], the frequency shift estimation in modern measurement systems for QCM sensors and other resonator-based bio- and chemical sensors must be performed in a reduced measurement time (less than 1/10 s).

The fully digital measuring system for study of the dynamic response of QCM sensors is described in [1]. The frequency shift is measured by a dedicated fully digital front-end hardware implementing a technique proposed in [10]. However, the system has a low accuracy with an uncertainty of $5 \mathrm{~Hz}$ in a range of $30 \mathrm{kHz}$ that corresponds to $0.017 \%$ relative error. The measurement time for this system is $20 \mathrm{~ms} \times 10=200 \mathrm{~ms}$ because of frequency shifts are measured each $20 \mathrm{~ms}, 10$ successive measurements are averaged to obtain a frequency sample. The uncertainty can be reduced only by increasing the measurement time via software programming [1] that is in contravention with the requirements for modern measuring and data acquisition systems for QCM sensors. The used method critical issue is also to have an almost uniform distribution of the initial phases of the streams. Some sufficient conditions to ensure the uniform distribution of the initial phases are provided in [10], and one of these is a specific relationship between the sampling frequency and measured frequency. But this relationship can be achieved only for the nominal frequency. As the frequency shift, even of about a few $\mathrm{Hz}$ from the nominal frequency, this condition is no more fulfilled, and the measurement system performance gets worse. In this case the error committed by the system is increased.

Another frequency measurement technique for quartz microbalance systems is described in [11]. It uses a period counting based on a D flip-flop mixer, directly applicable to quartz microbalances and quartz resonator-based sensors. The architecture of integrated period counting system is based on the indirect (period) measurement of a low frequency difference signal obtained by subtracting a high stability reference frequency from the unknown frequency to be measured. A digital mixer (D flipflop based) circuit is used to obtain frequency differencing, eliminating the need for an analog mixer and low-pass filters. Using a method of reducing the risk of miscloking in the digital mixer the expected error of such system can be $0.06 \mathrm{ppm}$. But this measure leads to significant input frequency range narrowing (up to $7.006 \div 7.030 \mathrm{MHz}$ ) because of it needs a proper selection of the minimum difference of input frequencies that can reduce the probability of misclocking events to insignificance.

Very often the standard desktop frequency counters are used in QCM sensors-based systems in order to measure a resonance frequency [2]. As usually frequency counter-based systems has absolute error $\pm 1 \mathrm{~Hz}$ at $1 \mathrm{~s}$ measuring time. Such solution is expensive, needs complex automatization for experiment and is not suitable for industrial systems of analytic chemistry.

\section{MODERN REQUIREMENTS TO DAQ SYSTEMS FOR QCM SENSORS}

Based on the state-of-the-art review of frequency measurement and data acquisition systems for QCM and other resonator-based bio- and chemical sensors it is possible to formulate main requirements to different modern gravimetric analyze-based systems. Firstly, all such systems must have low relative error 
of measurement (less than $0.001 \%$ ) constant in all frequency range, and high resolution. Secondly, the systems must have a possibility to measure both resonance frequency (sometimes more than the reference frequency) and quartz crystal quality factor $Q$; to determine a time constant for chemical transient and different dynamic behaviours; and to exploit a kinetic for adsorption processes.

All these require the reduced time of measurement (less than $0.1 \mathrm{~s}$ ). Such systems must have a wide frequency range without any restrictions for the input signal and let directly convert a frequency shift to digital without a preliminary conversion the unknown frequency to low frequency range. It should be also inexpensive and have simple interface electronics. At first sight some of requirements seem to be in contradiction but such problem was solved by the use of novel frequency measurement technique and integrated circuit on its basis.

\section{ADVANCED MODIFIED METHOD OF THE DEPENDENT COUNT}

The quartz microbalance system's efficiency is determined by the method for frequency measurements. For a high performance frequency measurement system designed for different QCM and other resonator-based bio- and chemical sensors it is expediently to use the proposed digital method for frequency shift measurements based on the modified method of the dependent count (MDC) [12-14].

In comparison with the patented earlier method [15], the modified MDC has an increased metrological reliability, and in comparison with the method [16] - an extended high frequency range and a possibility to measure unknown frequency $f_{x}$ in excess of the reference frequency: $f_{x}>>f_{0}$. In comparison with the methods described in $[12,15$, $16]$, new recently patented modified method in additional to the high programmable accuracy (for each of measurement if it is necessary according to a measuring algorithm), constant quantization error in all frequency range (from infralow up to high frequency), scalable resolution, non-redundant time of measurement and wide frequency range lets to measure frequency that is exceeds the reference frequency: $f_{x}>>f_{0}$ without additional hardware and time for preliminary frequency estimation or comparison: $f_{x}><f_{0}$. The time diagram of the modified MDC is shown in Figure 1.

Let consider two possible cases. In the case of $f_{x}<f_{0}$, where $f_{0}$ is the reference frequency; time diagrams of the method are similar to the proposed early the method of the dependent count [12] but without the preliminary stage for frequency comparison $\left(f_{x}><f_{0}\right)$, Figure 1 a.

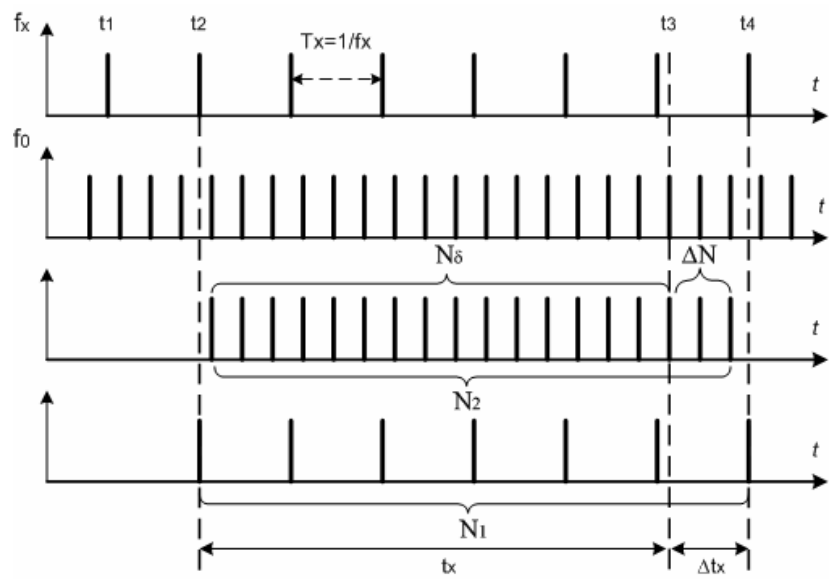

a)

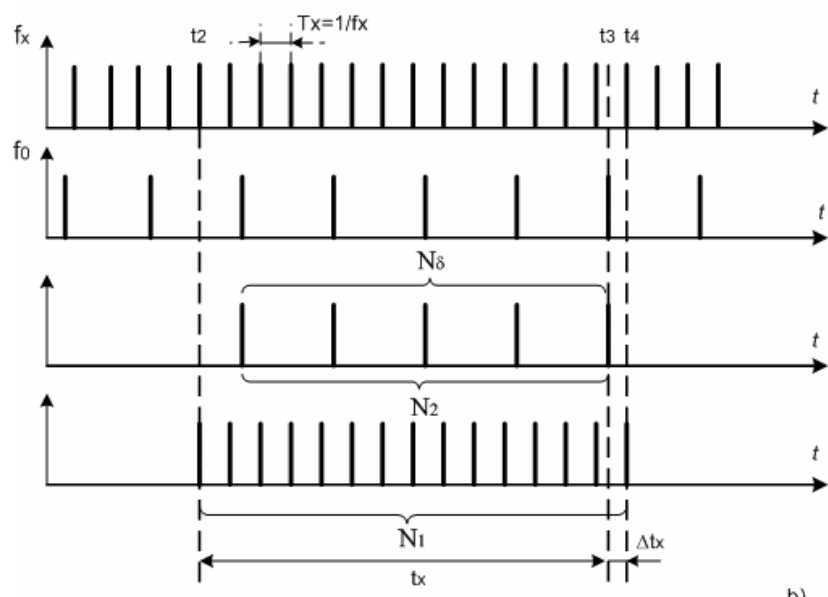

b)

Fig. 1 - Time diagrams of the modified method of the dependent count in case of $f_{x}<f_{0}$ (a) and $f_{x}>f_{0}$ (b).

In other words, a measurement will start immediately at the $t 2$ moment. Beginning from the moment $t 2$ according to the modified method, pulses of both frequencies $\mathrm{fx}$ and $\mathrm{f0}$ are counting (the numbers $\mathrm{N} 1$ and $\mathrm{N} 2$ respectively). The count is continuing during the time interval between $\mathrm{t} 2$ and t4. The reference gate time between $\mathrm{t} 2$ and $\mathrm{t} 3$ equals to the integer number of periods of the reference frequency $\mathrm{f} 0$ and is determined by the number $\mathrm{N} \delta=$ $1 / \delta$, where $\delta$ is the relative error of measurement. The necessary relative error can be chosen at the beginning of each of measurement or set up for all measurements at once according to a measuring algorithm. The counts of both frequencies are stopped by the next pulse of unknown frequency fx after the moment $t 3$. The number $\mathrm{N} 2$ is equals to the integer number of period of the reference frequency f0:

$$
N_{2}=N_{\delta}+\Delta N \text {, }
$$

where $\Delta N=\left(0 \div \Delta N_{\max }\right)$ is the additional number of 
period of the reference frequency $f_{0}$ counted during the $\Delta t$ time interval (between $t_{3}$ and $t_{4}$ ). In other turn, the number $N_{1}$ equals to the integer number of pulses of unknown frequency $f_{x}$.

The time of measurement $T_{\text {meas }}$ equals to an integer number of converted periods of the signal with frequency $f_{x}$ :

$$
T_{\text {meas }}=T_{x} \cdot N_{1}=\frac{N_{1}}{f_{x}},
$$

This time interval can also be given as:

$$
T_{\text {meas }}=T_{0} \cdot N_{2}=\frac{N_{\delta}+\Delta N}{f_{0}},
$$

From equations (2) and (3) it follows that unknown frequency should be calculated according to following formulas:

$$
f_{x}=\frac{N_{1}}{N_{2}} \cdot f_{0}
$$

The quantization error for these measurements results from the fact that the time of measurement $T_{\text {meas }}=t_{x}+\Delta t_{x}$ does not equal to an integer number $N_{2}$ of period of the reference frequency $T_{0}=1 / f_{0}$ :

$$
T_{\text {meas }} \neq N_{2} \cdot T_{0} \neq \frac{N_{2}}{f_{0}}
$$

A change of the frequency $f_{x}$ between specific limits will change the interval $T_{\text {meas }}$, and the number of counted impulses $N_{2}$ will be changed from $\Delta N=0$ to $\Delta N=\Delta N_{\max }$, where $\Delta N_{\max }$ is the number of impulses in the interval $\Delta t_{x} \max =T_{x}$. Taking into account that the period of these input impulses is $T_{X}$, we will have

$$
\Delta N_{\max }=\frac{T_{x}}{T_{0}}=\frac{f_{0}}{f_{x}}
$$

The maximal quantization error will take place when the number of counted impulses $N_{2}$ is minimal and equals to $N_{\delta}$. Then the maximal relative error will be

$$
\delta_{\text {max }}=\frac{1}{N_{\text {min }}}=\frac{1}{N_{\delta}}=\delta
$$

Hence, the maximal error is determined only by $\mathrm{N} \delta$ and does not depend on the measured frequency. The minimal error will be at $\mathrm{N}=\mathrm{Nmax}$. Since
$\mathrm{Nmax}=\mathrm{N} \delta+\Delta \mathrm{Nmax}$

$$
\delta_{\min }=\frac{1}{N_{\delta}+\Delta N_{\max }}
$$

Taking into account a wide frequency range of measurement the time of measurement Tmeas $=\mathrm{tx}+\Delta \mathrm{tx}$ should be calculated as:

$$
\left\{\begin{array}{l}
T_{\text {meas }}=T_{x}, \quad \text { if } \quad \frac{N_{\delta}}{f_{0}} \prec T_{x} \\
T_{\text {meas }}=\frac{N_{\delta}}{f_{0}}+\left(0 \div T_{x}\right), \text { if } \quad \frac{N_{\delta}}{f_{0}} \geq T_{x}
\end{array}\right.
$$

The resolution of method in the case of $\mathrm{fx}<\mathrm{f0}$ can be calculated according to the following equation:

$$
Q_{N}=\frac{f_{0} \cdot N_{1}}{N_{2}\left(N_{2}+1\right)}=\frac{f_{x}}{\left(N_{2}+1\right)}=\frac{f_{x}}{\left(N_{\delta}+\Delta N+1\right)}
$$

In the case of $f_{x}>f_{0}$ time diagrams of the method are shown in Figure $1 \mathrm{~b}$. The number $N_{2}$ equals to the number $N_{\delta}(\Delta N=0)$. Unknown frequency $f_{x}$ should be calculated according to the same equations (5). The relative error is $\delta=\delta_{\max }=\delta_{\min .}$. The time of measurement in this case will be

$$
T_{\text {meas }}=\frac{N_{\delta}}{f_{0}}+\left(0 \div T_{x}\right)
$$

The resolution of modified method of the dependent count in case of $\mathrm{fx}>\mathrm{f0}$ can be determined as:

$$
Q_{N}=\frac{f_{x}}{\left(N_{\delta}+1\right)}
$$

The modified MDC lets to measure a frequency shift $\Delta \mathrm{fx}$ and a frequency deviation (absolute and relative). It means simple addition calculations according to the common equation [13]:

$$
R=\frac{a \cdot f_{x} \pm b}{c},
$$

where $a, b$ and $c$ are constants, which should be set up in the same unit as a measurand. So, at frequency deviation measurement, $a=0, c=0$ and

$$
\Delta f_{x}=f_{x}-b=\frac{n}{N} \cdot f_{0}-b,
$$


where the constant $b$ should be the frequency of reference quartz resonator in the case of QCM systems.

\section{HIGH PERFORMANCE, LOW-COST INTELLIGENT DAQ SYSTEM}

A developed data acquisition system for differential self-excited QCM sensors is shown in Figure 2 [17].

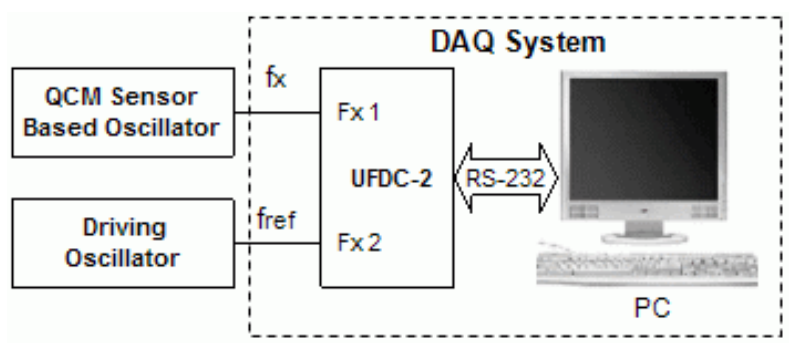

Fig. 2 - Intelligent data acquisition system for differential self-excited QCM sensors [17].

It is based on the designed integrated circuit of two-channel Universal Frequency - to - Digital Converter (UFDC-2). In comparison with the UFDC-1 [18], which is produced since 2004, the new integrated circuit has a special measuring mode for frequency deviation and extended frequency range up to $9 \mathrm{MHz}$ (or $144 \mathrm{MHz}$ with prescaling). It is based on the modified MDC and can measure frequency shift and deviation with the programmable relative error from 1 to $0.0005 \%$ and non-redundant measurement time. The quantization error is not dependent on the measurand frequency. It is constant in the whole frequency range and less than programmable relative error $\delta_{x}<\delta$. The UFDC-2 has a quartz-accurate automated calibration mode; RS-232 (422/485), SPI and $\mathrm{I}^{2} \mathrm{C}$ interfaces; master and slave communication modes. The reference frequency $f_{0}=625 \mathrm{kHz}$ for a basic IC version and $20 \mathrm{MHz}$ for high speed IC modification. Intelligent (smart) UFDC-2 features include a self-adaptation possibility (a flexibility to change accuracy for speed and opposite during each of measurement). A custom design version of UFDC-2 can also store an IEEE 1451 Transducer Electronic Data Sheets (TEDS) in its flash memory with the aim to simple QCM sensor configuration in a different systems and self-identification purpose.

In the data acquisition systems based on the UFDC-2 any differential QCM or resonator-based sensor can be connected to the first IC's channel, and the driving oscillator - to the second channel. For such connections, the UFDC-2 should be used in a frequency difference measurement mode $\left(f_{x 1}-f_{x 2}\right)$ with a sign determination. In this case $f_{x}=f_{x 1}$ and $f_{\text {ref }}=f_{x 2}$. The sign "minus" before the result of measurement testifies that $f_{x 1}<f_{x 2}$. The range of possible programmable relative error of measurement is from 1 to $0.0005 \%$. The mode for frequency difference measurement with prescaling (external divider by 16) is also possible.

Data exchange between the measuring system and PC computer can be established by the serial interface RS-232 and any terminal window software, for example, freeware software Terminal v.1.9b for Windows. Naturally, the LabVIEW software from National Instruments (USA) can be also used in such systems to establish a communication between the UFDC-2 and PC computer as well as design different virtual instruments for QCM or any resonator-based sensors. Appropriate commands (at RS-232 interface communication), which should be typed in the terminal window for the UFDC-2 working in the frequency shift measuring mode are shown in Figure 3.

$\begin{array}{ll}\text { >M06 } & \text {; Frequency difference measurement initialization } \\ \text { >A0A } & \text {; } 0.0005 \% \text { conversion relative error set up } \\ \text { >S } & \text {; Start a measurement } \\ \text { >R } & \text {; Read a result } \\ 7054.07537 & \text {; Measurement result indication }\end{array}$

Fig. 3 - UFDC-2 commands for working in the frequency difference measuring mode $\left(f_{x 1}-f_{x 2}\right)$.

Based on the received frequency shift measurement result a computer should calculate the appropriate mass and volume of chemical agent. The DAQ board prototype is shown in Figure 4.

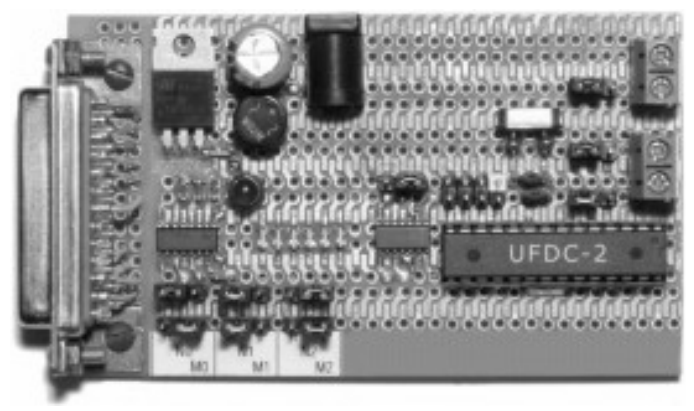

Fig. 4 - Prototype of DAQ board.

In order to measure a frequency shift with the programmable relative error batter than $0.01 \%$, the UFDC-2 should be calibrated before. The IC provides so-called in-circuit quartz-accurate automated calibration. The calibration is made by means of the communication interface RS-232. The calibration sequence for the UFDC-2 is the following. The test command "T" toggles the IC to the calibration mode. A sequence of rectangular pulses with frequency $f_{\text {osd }} / 2$ is generated at the TEST output. The frequency of this pulse sequence should 
be measured with the help of an external frequency counter with accuracy not worse than $0.00005 \%$ or, at least, $0.0001 \%$. The command "Fi.f" will put the clock constant value into the UFDC-2. The calibration should be performed only once. The correction factor $\Delta$ is stored in the nonvolatile memory of the UFDC-2, which holds this value after power-off.

In some measuring systems instead of the driving oscillator, the numerical reference value of its frequency $f_{\text {ref }}$ can be used. It this case, the UFDC-2 should be working in the absolute frequency deviation measuring mode to measure the frequency shift $\Delta f=f_{x 1}-f_{\text {ref. }}$. An example of such system is shown in Figure 5 [17].

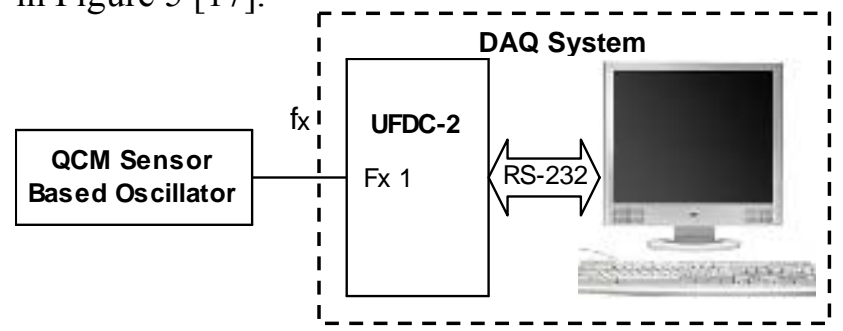

Fig. 5 - Data acquisition system for QCM sensor [17].

Appropriate commands for the UFDC-2 working in the absolute frequency deviation measuring mode are shown in Figure 6.

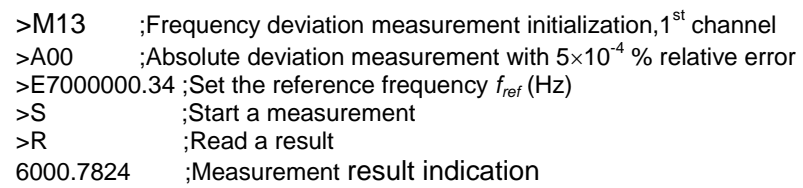

Fig. 6 - UFDC-2 commands for working in the absolute frequency deviation measuring mode $\left(f_{x 1}-f_{\text {ref }}\right)$.

\section{MODELING AND EXPERIMENTAL RESULTS}

The modelling result for time of measurement at relative error of measurement $\delta \in[0.01 \div$ 0.000005], reference frequency $f_{0}=625 \mathrm{kHz}$ (a), $f_{0}=20 \mathrm{MHz}$ (b) and measuring frequency range $f_{x} \in$ [0.8 $\div 9] \mathrm{MHz}$ is shown in Figure 8 accordingly. As it is visible from the plot, time of measurement does not depend on the measurand frequency $f_{x}$ and frequency difference in the high frequency range. It is non-redundant and mainly determined by the programmable relative error of measurement $\delta$.

The objective of experimental investigations was to determinate metrological performances for UFDC-2 at precise frequency measurements in two channels correspond to measurements for differential self-excited QCM sensors. The measurement set up is shown in Figure 9.
A square waveform pulse signal with frequency $f_{x 1}=9000000.211 \mathrm{~Hz}$ corresponds to a driving oscillator's fundamental frequency was generated by the first functional generator Agilent 33220A and measured in the $2^{\text {nd }}$ cannel $\mathrm{FX}_{2}$ of UFDC-2; and square waveform pulse signal with frequency $f_{x 2}=8999800.078 \mathrm{~Hz}$ corresponds to a QCM sensor based oscillator change in resonant frequency $\left(\Delta f=f_{x 1-} f_{x 2}\right)$ in $\mathrm{Hz}$ is directly proportional to the mass adsorbed on the crystal surface was generated by the second functional generator Agilent 33220A

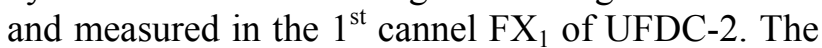
IC was programmed to measure frequency with the minimal possible relative error $0.0005 \%$. The supply voltage of the evaluation board was $+5 \mathrm{~V} \mathrm{dc}$, provided by the Promax FAC-363B power supply. Both frequencies were controlled by a frequency counter Agilent 53132A with ultra high oven stability internal time base. The two-channel digital oscilloscope Promax OD-571 monitored the signals waveforms. Before measurements, the UFDC-2 was calibrated in the working temperature range: $+22.7 \ldots+23.9^{\circ} \mathrm{C}$.

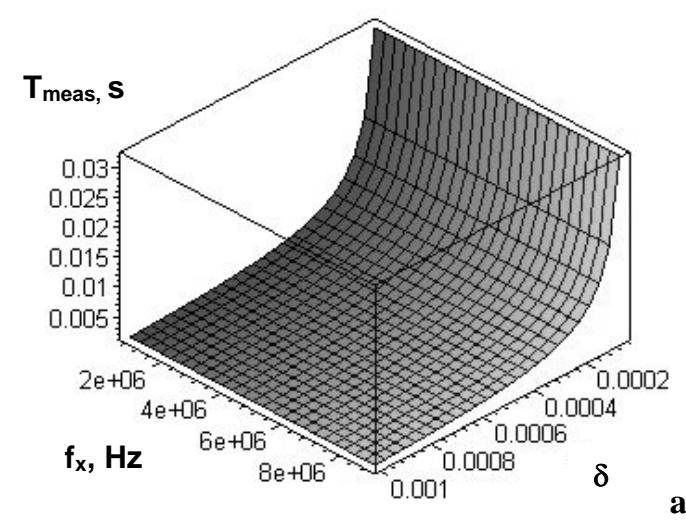

a)

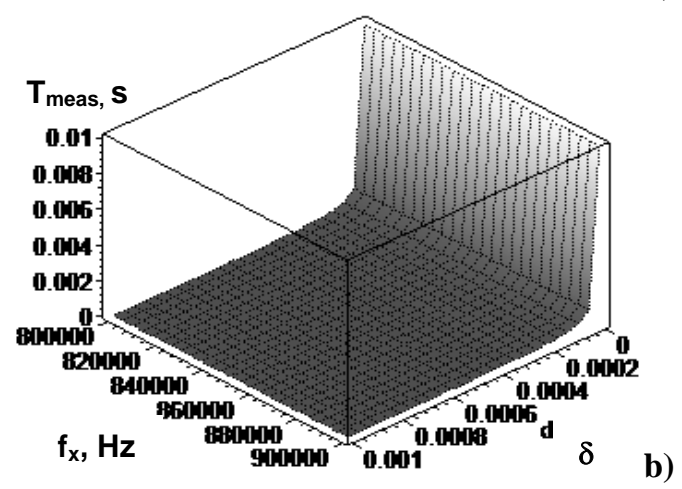

Fig. 8 - The modelling result for time of measurement $T_{\text {meas }}=f\left(f_{x}, \delta\right)$ at $f_{0}=625 \mathrm{kHz}(\mathrm{a})$ and $f_{0}=20 \mathrm{MHz}(\mathrm{b})$.

The measured values were sent to a $\mathrm{PC}$ via an RS-232 (USB) interface implemented with the ST202D IC. The user interface was realized with the help of terminal software Terminal $V 1.9 b$ for Windows XP. Each of measurements was taken until 
totalling 32 values. The measurement errors were evaluated from appropriate statistics. Results of frequency measurements for driving and QCM sensor based oscillator are shown in Figure 10. The statistical characteristics are adduced in Table 1 and appropriate densities of distributions are shown in Figure $11(\mathrm{a}, \mathrm{b})$. As it is visible from the table, the maximal relative error does not exceed the programmable $\delta_{x}<0.0005 \%$.

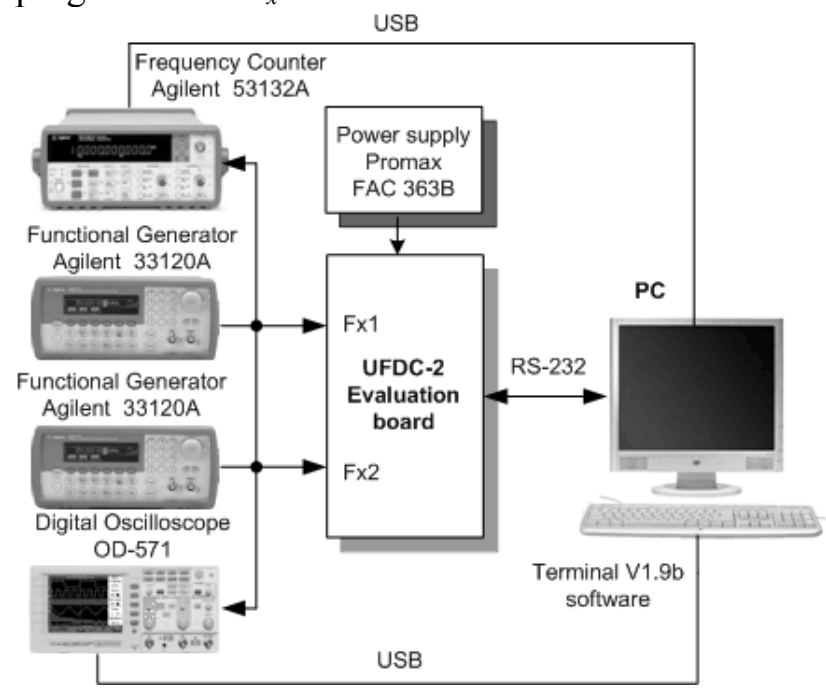

Fig. 9 - Measurement set up.

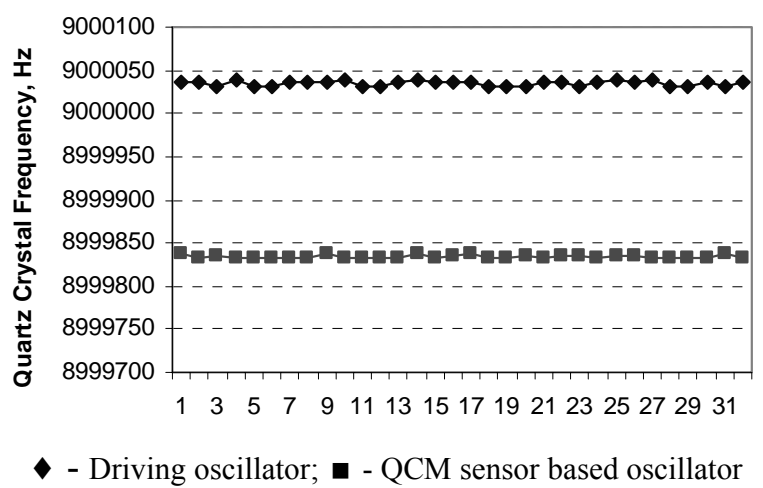

Fig. 10 - Measurements results for driving and QCM sensor based oscillator.

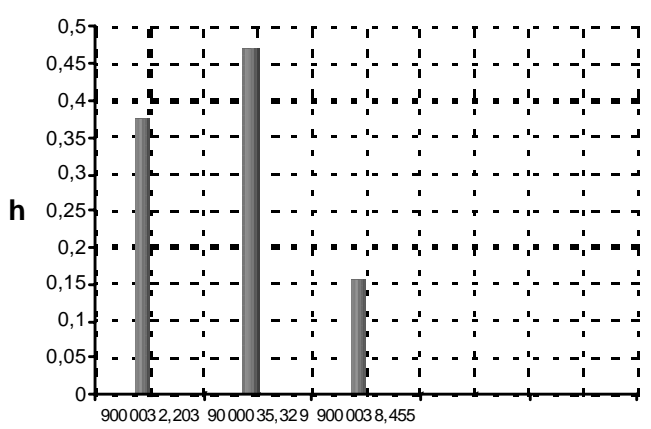

Frequency, $\mathrm{Hz}$

a)

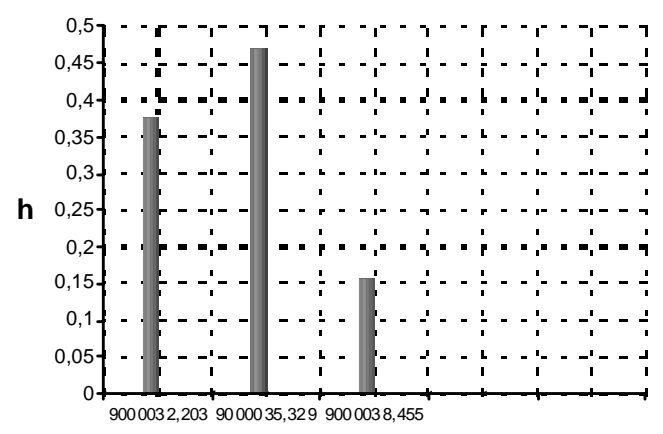

Frequency, $\mathrm{Hz}$

b)

Fig. 11 - Density of distribution for driving (a) and QCM sensor based (b) oscillators.

Table 1. Statistical Characteristics.

\begin{tabular}{|l|l|l|}
\hline \multicolumn{1}{|c|}{ Parameter } & \multicolumn{2}{c|}{ Value } \\
\hline $\begin{array}{l}\text { Number of } \\
\text { measurements, } N\end{array}$ & 32 & 32 \\
\hline Minimum $f_{x}(\mathrm{~min}), \mathrm{Hz}$ & 9000032.2 & 8999832.15 \\
\hline $\begin{array}{l}\text { Maximum } f_{x}(\max ), \\
\text { Hz }\end{array}$ & 9000038.45 & 8999838.4 \\
\hline $\begin{array}{l}\text { Sampling range, } \\
f_{x}(\max )-f_{x}(\min ), \mathrm{Hz}\end{array}$ & 6.2515 & 6.2515 \\
\hline Median & 0 & 0 \\
\hline Arithmetic mean, Hz & 9000034.64 & 8999833.81 \\
\hline Variance & 4.8753 & 5.6633 \\
\hline Standard deviation & 2.208 & 2.3798 \\
\hline $\begin{array}{l}\text { Coefficient of } \\
\text { variation }\end{array}$ & 4076067.12 & 3781815.29 \\
\hline $\begin{array}{l}\text { Confidence interval at } \\
\text { probability } P=97 \%\end{array}$ & $\begin{array}{l}f_{x} \epsilon \\
{[9000033.79 \div} \\
9000035.49]\end{array}$ & $\begin{array}{l}f_{x} \epsilon \\
{[8999832.9 \div} \\
8999834.72]\end{array}$ \\
\hline Relative error, \% & $\begin{array}{l}0.00036< \\
0.00050\end{array}$ & $\begin{array}{l}0.00043< \\
0.00050\end{array}$ \\
\hline
\end{tabular}

In comparison with the frequency measurement system described in [9], the proposed system at the same relative error of measurement $\delta=0.005 \%$ and frequency range lets receive the time of measurement $32 \mathrm{~ms}$ in comparison with $100 \mathrm{~ms}$ reported in [9]. In comparison with the frequency measurement system based on the all-digital frequency detector described in [1], the proposed system at the same relative error of measurement $\delta=0.01 \%$ and frequency range lets receive the time of measurement $16 \mathrm{~ms}$ in comparison with $20 \times 10=200 \mathrm{~ms}$ reported in [1], where frequency shifts are measured each $20 \mathrm{~ms}$ and 10 successive measurements are averaged to obtain a frequency sample.

The same time of measurement has also the integrated frequency measurement system based on the FPGA XC3142A from Xilinx [19]. It uses the 
classical indirect method for period measurement. This system was set up to take 250 periods for the measurement, which is close to the maximum allowed at the oscillator frequency of $1.23 \mathrm{kHz}$ this equates to a measurement time of around $200 \mathrm{~ms}$.

The theoretical error in this system is significantly lower than the experimental results due to the noise in the system, which needs to be improved. Such property belongs to any system based on the period measurements.

In comparison with the frequency measurement technique described in [11], the strict requirements to the input signal, which limited its frequency range, are absent in the proposed measuring system. The frequency range can be from $0.05 \mathrm{~Hz}$ to $9 \mathrm{MHz}$ (without prescaling) at constant quantization error in all frequency range, which does not exceed the programmable relative error of measurement and can be chosen, for example, $0.0005 \%$ that corresponds

5 ppm.

All these made the proposed frequency measurement system very suitable for QCM or resonator-based sensor interfacing in liquids and gases. Due to its non-redundant and reduced time of measurement the developed system can be used for a chemical transient time constant and different dynamic behaviours determination in order to exploit a kinetic for adsorption processes. Such kind of low-cost frequency measuring systems are based on the UFDC-2 can be used in full system-on-chip electronic noses and tongues.

This approach has eliminated much of the hardware associated with most measuring systems for QCM sensors; there is no need for a microcontroller in the system and complex sensor interfacing electronics as it is directly compatible with an IBM PC compatible computers and use only one IC.

\section{CONCLUSIONS}

The data acquisition system for QCM and other (superficial SAW and bulk acoustic wave) resonator-based chemical and biosensors described in this article is based on the novel frequency measurement technique using the modified method of the dependent count and allows obtaining high metrological performances: programmable, constant in all frequency range, relative error of measurement up to $0.0005 \%$ that corresponds to $5 \mathrm{ppm}$; nonredundant, reduced conversion time of measurement and wide frequency range from $0.05 \mathrm{~Hz}$ to $9 \mathrm{MHz}$ (144 MHz with prescaling). Due to all these the developed system can be used for a chemical transient time constant and different dynamic behaviours determination in order to exploit a kinetic for adsorption processes.

The system satisfies to all modern requirements and can measure both resonance frequency (sometimes more than the reference frequency) and quartz crystal quality factor $Q$ for QCM sensors working, for example, in liquids and gases.

Described low-cost data acquisition system is based on the integrated circuit of universal frequency-to-digital converter (UFDC-2). This design approach has eliminated much of the hardware associated with most measuring systems for QCM sensors; there is no need for a microcontroller in the system and complex sensor interfacing electronics as it is directly compatible with an IBM PC compatible computers and use only one IC that can be used in industrial full system-onchip electronic noses and tongues.

\section{ACKNOWLEDGEMENTS}

This research was supported by the EC Marie Curie Chair (EXC) grant in frame of project MEXT-CT-2005-023991 Smart Sensors Systems (SMARTSES).

\section{REFERENCES}

[1] C. Di Nucci, A. Fort, S. Rocchi, L. Tondi, N. Uliviery, V. Vignoli, F. Di Francesco, M. B. Serrano-Santos, Study of the Dynamic Response of QCM sensors my means of a fast and accurate all-digital frequency detector. Proceedings of "IEEE Instrumentation and Measurement Technology Conference", Anchorage, USA, 2002, (CD-ROM).

[2] M. T. S. R. Gomes, Bulk acoustic wave sensors in chemical analysis. Smart Sensors and MEMS, S. Y. Yurish, M. T. S. R. Gomes, Ed. , Springer Verlag, 2005, pp.421-445.

[3] R. Lucklum, R. Borngraeber, P. Hauptmann, Acoustically amplified chemical QCM-sensor with frequency and attenuation output. Proceedings of “Transducers '99”, Sendai, Japan, 1999, pp.1250-1253.

[4] Hatfield, A. R. Daniels, D. Snowden, K. C. Persaud, P. A. Payne. Development of a hand held electronic nose (H2EN). Proceedings of "European Conference on Solid-State Transducers" (EUROSENSORS XIII), The Hague, The Netherlands, 1999, pp. 215-218.

[5] Inacio, J.N. Marat-Mendes, C. J. Dias. Development of a biosensor based on a piezoelectric film. Proceedings of "European Conference on Solid-State Transducers" (EUROSENSORS XVI), Prague, Czech Republic, 2002, vol.3, pp.763-764.

[6] E. Katz. Electronic biosensors based on biomaterial-nanoparicle hybrid systems. Smart 
Sensors and MEMS, S. Y. Yurish, M. T. S. R. Gomes, Ed., Springer Verlag, 2005, pp.447-474.

[7] S. R. Suri. Quartz crystal based microgravimetric immunobiosensors. Sensors \& Transducers, (66) (2006), pp. 544-552.

[8] S. Kurosawa, H. Aizawa, M. Tozuka, M. Nakamura, J.-W. Park. Immunosensors using a quartz crystal microbalance, Measuring Science and Technology, (14) (2003), pp. 1882-1887.

[9] L. A. Kuznetsov, M. V. Milonov, D. V. Nalivkin. A method for high-precision measurements of vibration frequency of piezoelectric sensors. Sensors \& Systems, February 2004 pp.2-6 (in Russian).

[10] M. T. Hill, A. Cantoni. Precise all-digital frequency detector for high frequency signals. IEEE Transactions on Communications, (48) (2000), pp.1937-1944.

[11] Shankar, S.A. Morris, C.G. Hutchens. A novel measurement technique for quartz microbalance systems and other resonator-based sensor systems. Proceedings of "Sensors for Industry Conference (Sicon/02)”, Houston, Texas, USA, 2002, pp.134-138.

[12] N. V. Kirianaki, S. Y. Yurish, N. O. Shpak. Methods of dependent count for frequency measurements, Measurement, (29), 2001, pp.31-50.

[13] N. V. Kirianaki, S. Y. Yurish, N. O. Shpak, V. P. Deynega. Data Acquisition and Signal Processing for Smart Sensors. Chichester, UK: John Wiley \& Sons, 2001.

[14] N. V. Kirianaki, S. Y. Yurish, Method of measurement for frequency and period of harmonic signal and device for its realization, UA Patent No. 81851, G01R 23/02, February 2008.

[15] N. V. Kirianaki, B. M. Berezyuk. Method of measurement for frequency and period of harmonic signal and device for its realization, USSR Patent 788018, December 1980.

[16] L. A. Vinogor, N. V. Kirianaki. Device for frequency and period measurement of harmonic signal, Russia Patent 1797714, February 1993.

[17] Yurish S. Y., Intelligent Data Acquisition System for Quartz Crystal Microbalance and Other Resonator-Based Sensors. Proceedings of IEEE International Workshop on Intelligent
Data Acquisition and Advanced Computing Systems: Technology and Applications, 6-8 September 2007, Dortmund, Germany, pp. 2-7.

[18] S. Y. Yurish, N. V. Kirianaki, R. Pallas-Areny, Universal frequency-to-digital converter for quasi-digital and smart sensors: specification and applications, Sensor Review, 25 (2) (2005), pp. 92-99.

[19] R. Stone, P.A. Payner. Measurement of the response of resistive sensors using logic oscillators and integrated frequency measurement, Sensors and Actuators A: Physical, 76 (1-3) (1999), pp.431-436.

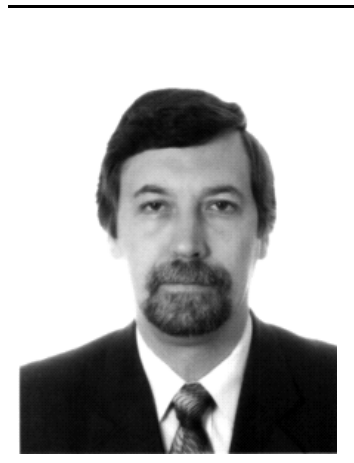

Sergey $Y$. Yurish Received his PhD degree in Measuring Instruments and Methods for Measuring Electrical and Magnetic Quantities from National University Lviv Polytechnic in 1997. He holds 9 patents, has published 4 books and more than 130 scientific papers published in periodicals and conference proceedings. Since 2006 he is a Marie Curie investigator in Technical University of Catalonia (UPC-Barcelona). His research interests include methods for precise frequency-time measurements, integrated frequency-to-digital converters, intelligent data acquisition systems, direct sensor-to-microcontroller interfaces, smart sensors, transducers and sensor systems. 